\title{
Analysis of vascular function using the cardio-ankle vascular index (CAVI)
}

\author{
Kohji Shirai \\ Hypertension Research (2011) 34, 684-685; doi:10.1038/hr.2011.40
}

\section{THE ROLES OF SMOOTH MUSCLE CONTRACTION IN CAVI}

Tsing the cardio-ankle vascular index (CAVI), Kim et al. ${ }^{1}$ studied the hemodynamics of subjects under general anesthesia and published the results in Hypertension Research. The CAVI was developed as a new index of the stiffness of the artery as a whole, from the origin of the aorta to the ankle. ${ }^{2-4}$ The authors observed that both systolic and diastolic blood pressure decreased during generalized anesthesia using propofol. Simultaneously, brachial-ankle pulse wave velocity decreased along with the decrease in blood pressure. In contrast, CAVI did not decrease, and no correlation between CAVI and changes in blood pressure was observed. The authors concluded that CAVI is a useful index of true arterial stiffness and is superior to brachial-ankle pulse wave velocity. This conclusion might be reasonable. However, the data in Table $2^{1}$ show that CAVI also decreased by $7.6 \%$ during anesthesia in persons $<65$ years old, and no correlation between CAVI and blood pressure was observed. This finding should not be ignored for the following reason: General anesthesia using propofol, which has a sedative effect and a vasodilator action, ${ }^{5}$ might have some effects on the tone of arterial wall smooth muscle cells. Under general anesthesia, it is probable that the activity of the sympathetic nerves that control vasoconstriction might be depressed, in which case the peripheral arteries will be dilated or relaxed. Blood pressure will decrease as a result. In addition, brachial-ankle pulse wave velocity will thus

Correspondence: Dr K Shirai is at the Department of Internal Medicine, Sakura Hospital, School of Medicine, Toho University, 564-1 Shimoshizu, Sakura-shi, Chiba 285-8741, Japan.

E-mail: kshirai@kb3.so-net.ne.jp decrease because pulse wave velocity depends on blood pressure at the time of the measurement, as reported by Nye et al. ${ }^{2-4,6}$ Under these conditions, a decrease in CAVI might be due to the relaxation of smooth muscle cells induced by general anesthesia itself and by administration of propofol. The report by Kim described two important features of CAVI: (1) CAVI expression changes over a short period of time, depending on the circulatory condition; and (2) CAVI expression reflects the state of smooth muscle cell contraction.

Here, another question was raised. Why was the CAVI value not reduced significantly in patients $>65$ years old during general anesthesia? Notably, the CAVI decreased from 9.30 to 8.95 . The CAVI in this group ( $>65$ years old) was significantly higher than that of the group $<65$ years old. Therefore, the arterial wall in patients $>65$ years old with a high CAVI could not relax to a sufficient extent because sclerosis of the arterial wall was advanced compared with that in patients $<65$ years old.

We previously showed that the CAVI represents both organic and functional stiffness, based on smooth muscle contraction. ${ }^{4}$ The paper demonstrated the effect of beta- and alpha-blockers on the CAVI in humans. In this study, a selective $\beta 1$-receptor blocker, metoprolol, which is known to reduce the contraction of the heart muscle and decrease blood pressure without affecting the tone of the arterial wall, was administered to 12 men. Over the course of $6 \mathrm{~h}$, brachial-ankle pulse wave velocity decreased, but the CAVI did not change. This result clearly demonstrates that the CAVI was not influenced by the blood pressure at the time of measurement.

Furthermore, the $\alpha 1$-adrenergic receptor blocker doxazosin induces the relaxation of smooth muscle cells, and thereby, dilates the peripheral arteries and decreases the blood pressure. $^{4}$ The CAVI decreased after the administration of doxazosin. These results support the hypothesis that the CAVI reflects vascular tone, representing both smooth muscle contraction and inorganic matrix components.

Thus, the value of the CAVI as a marker of vascular stiffness should be re-considered. CAVI is not merely a marker of aging or arteriosclerosis of the artery.

\section{CAN THE CAVI REFLECT VASCULAR FUNCTION?}

The CAVI is a new index of the stiffness of arteries of substantial length. ${ }^{2}$ The most conspicuous feature of the CAVI is lack of dependence on blood pressure at the time of measurement. This lack of dependence was predicted by theory and was confirmed by several experimental studies. ${ }^{2-4}$

The CAVI is high in aging patients and in patients with arteriosclerotic diseases, such as coronary artery disease, ${ }^{7}$ carotid arteriosclerosis, ${ }^{3,8}$ chronic kidney disease ${ }^{9}$, and is related to many coronary risk factors such as hypertension, ${ }^{3}$ diabetes mellitus, ${ }^{8}$ dyslipidemia $^{8}$ and smoking. ${ }^{10}$ Furthermore, the CAVI decreases with control of diabetes mellitus and hypertension, as well as with abstention from smoking. ${ }^{10}$ These factors suggest that the CAVI is a surrogate marker of arteriosclerosis.

In addition to those roles of the CAVI, Kim et al. ${ }^{1}$ reported that the CAVI could also reflect smooth muscle contraction. Thus, the CAVI might reflect the real stiffness of the artery. This concept provides new insight into the role of the systemic circulation. The circulatory system is composed of the heart, large- and medium-sized arteries and 

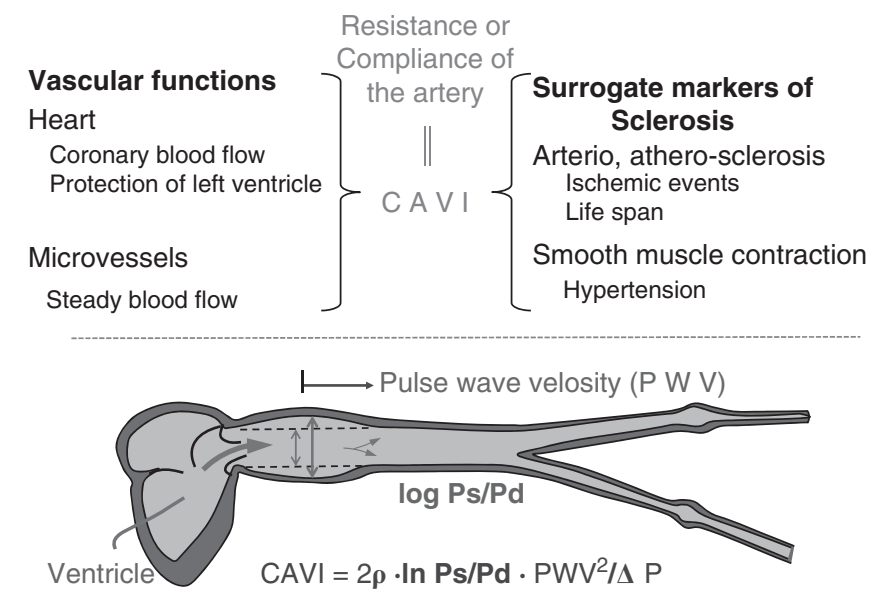

Figure 1 Cardio-ankle vascular index (CAVI) and its measurement. With the patient lying supine, electrocardiogram and heart sound are monitored. Pulse wave velocity from the heart to the ankle is obtained by measuring the length from the origin of the aorta to the ankle. Blood pressure is measured at the brachial artery. Ps, systolic blood pressure, $\mathrm{Pd}$, diastolic blood pressure, $\Delta \mathrm{P}, \mathrm{Ps}-\mathrm{Pd}, \rho$, blood density. CAVI could be not only a surrogate markers of arteriosclerosis, but also that of smooth muscle contraction. Furthermore, CAVI might be related with heart function and microvessel circulation.

microvessels. Pulsatile movement of the heart efficiently transports blood to the peripheral organs with the aid of vascular function; that is, the arteries dilate during the systolic phase and contract during the diastolic phase. Although this windkessel action has been ascribed to vascular compliance or resistance, an index to reflect this function was not previously available. The CAVI could be used to assess this function (Figure 1). The potential utility of the CAVI for analysis of the systemic circulatory system requires further investigation.

In summary, the possibility that the CAVI is not only a surrogate marker of arteriosclerosis but also a marker of peripheral resistance or compliance was effectively raised by this paper by Kim.
1 Kim B, Takada K, Oka S, Misaki T. Influence of blood pressure on cardio-ankle vascular index (CAVI) examined based on percentage change during general anesthesia. Hypertens Res 2011; 34: 779-783.

2 Shirai K, Utino J, Otsuka K, Takata M. A novel blood pressure-independent arterial wall stiffness parameter; cardio-ankle vascular index (CAVI). J Atheroscler Thromb 2006; 13: 101-107.

3 Takaki A, Ogawa H, Wakeyama T, Iwami T, Kimura M, Hadano Y, Matsuda S, Miyazaki Y, Matsuda T, Hiratsuka A, Matsuzaki M. Cardio-ankle vascular index is a new noninvasive parameter of arterial stiffness. Circ J 2007; 71: 1710-1714.

4 Shirai K, Song M, Suzuki J, Kurosu T, Oyama T, Nagayama D, Miyashita Y, Yamamura S, Takahashi M. Contradictory effects of $\beta 1$ - and $\alpha 1$-aderenergic receptor blockers on cardio-ankle vascular stiffness index (CAVI) - the independency of CAVI from blood pressure. J Atheroscler Thromb 2011; 18: 49-55.

5 Bentley GN, Gent JP, Goodchild CS. Vascular effects of propofol: smooth muscle relaxation in isolated veins and arteries. J Pharm Pharmacol 1989; 41: 797-798.

6 Nye ER. The effect of blood pressure alteration on the pulse wave velocity. Br Heart J 1964; 266: 261-265.

7 Nakamura K, Tomaru T, Yamamura S, Miyashita Y, Shirai K, Noike H. Cardio-ankle vascular index is a candidate predictor of coronary atherosclerosis. Circ $J$ 2008; 72: 598-604

8 Ibata J, Sasaki H, Kakimoto T, Matsuno S, Nakatani M, Kobayashi M, Tatsumi K, Nakano Y, Wakasaki H, Furuta $\mathrm{H}$, Nishi M, Nanjo K. Cardio-ankle vascular index measures arterial wall stiffness independent of blood pressure. Diabetes Res Clin Pract 2008; 80: 265-270.

9 Nakamura K, liduka T, Takahashi M, Shimizu S, Mikamo H, Nakagami T, Suzuki M, Hirano K, Sugiyama Y, Tomaru T, Miyashita Y, Shirai K, Noike H. Association between cardio-ankle vascular index and serum cystain c levels in patients with cardiovascular risk factor. J Atheroscler Thromb 2009; 16: 371-379.

10 Noike H, Nakamura K, Sugiyama Y, lizuka T, Shimizu K, Takahashi M, Hirano K, Suzuki M, Mikamo H, Nakagami T, Shirai K. Changes in cardio-ankle vascular index in smoking cessation. I Atheroscler Thromb 2010; 17: 517-525. 